\title{
Real-Time Classification of Hand Motions Using Electromyography Collected from Minimal Electrodes for Robotic Control
}

\author{
Richard Byfield*1, Richard Weng ${ }^{1}$, Morgan Miller ${ }^{1}$, Yunchao Xie ${ }^{1}$, Jheng-Wun Sü ${ }^{2}$ Jian Lin ${ }^{1,3}$ \\ ${ }^{1}$ Department of Mechanical and Aerospace Engineering, University of Missouri, Columbia, Missouri, USA \\ ${ }^{2}$ Department of Physics and Engineering, Slippery Rock University, Slippery Rock, Pennsylvania, USA \\ ${ }^{3}$ Department of Electrical Engineering and Computer Science, University of Missouri, Columbia, Missouri, USA
}

Received: June 21, 2021

DOI: $10.5430 /$ ijrc.v3n1p13
Accepted: July 12, 2021

Online Published: August 2, 2021

\begin{abstract}
In recent years, advances in human robot interaction (HRI) has shown massive potential for universal control of robots. Among them, electromyography (EMG) signals generated by motions of muscles have been identified as an important and useful source. Powered by recently emerged machine learning algorithms, real-time classification has been proved applicable to control robots. However, collecting EMG signals with minimum number of electrodes for real-time classification and robotic control is still a challenge. In this paper, we demonstrate that twenty five robotic commands in a robotic arm can be controlled in real time by using the EMG signals collected from only two pairs of active surface electrodes on each forearm of human subjects. To achieve this task, a variety of tested ML models for this classification were tested. Among them, the Gaussian Naïve Bayes (GNB) achieved an accuracy of $>96 \%$. This unprecedented level of classification accuracy of the EMG signals collected from the least number of active electrodes suggest that by combination of optimized electrode configuration and a suitable ML model, the capability of robotic control can be maximized.
\end{abstract}

Key Words: EMG, Robots, Real-time classification, Machine learning

\section{INTRODUCTION}

Electromyography (EMG) signals are generated by the muscle motions and controlled by the nervous system. Recently, research of using EMG signals as a control source for robots has emerged due to a variety of potential applications. They can be used to control prosthetic devices, such as lower body prosthetics $^{[1,2]}$ and upper body prosthetics ${ }^{[3-5]}$ for amputees and partially paralyzed individuals, as the signals can directly reflect the users' motion intention. Incorporation of EMG signals in the control loop for electric wheelchairs manipulation enhances the capability and intelligence of the wheelchairs. ${ }^{[6,7]}$ Researchers have also explored using EMG signals to control exoskeletons. ${ }^{[8-10]}$ Control of robots through EMG signals in a factory setting can also greatly increase the adaptability of the robots, thus promoting the productivity.

The research of using EMG signals to control robots leads to an important paradigm of human-robot interaction (HRI) (see Figure 1). A HRI system via EMG starts from collection of EMG from human's muscles. The accuracy and consistency

*Correspondence: Richard Byfield; Email:rsbggb@umsystem.edu; Address: Department of Mechanical and Aerospace Engineering, University of Missouri, Columbia, Missouri 65211, USA. 


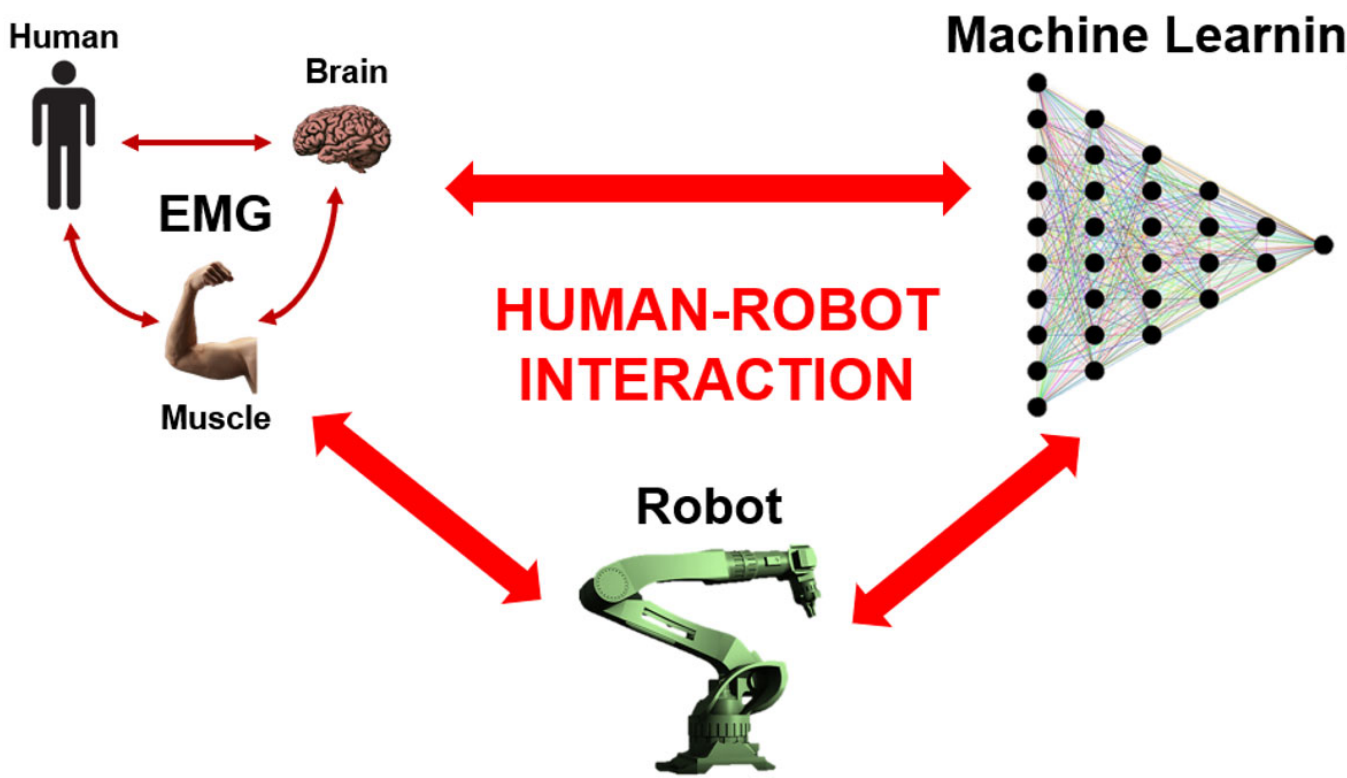

Figure 1. General overview of human robot interaction via EMG

of the EMG signals are largely influenced by the testing subjects, testing sites, and skin surface geometries. Increasing the number of electrodes used for testing may mitigate such an issue, but it inevitable increases the complexity of signal processing, thus imposing delay to the system control. After the signal collection, the signal preprocessing is needed to extract the feature sets from the EMG signals for later modeling or classification, which can discriminate the feature sets for different types of motions. Recently emerged data driven approaches enabled by machine learning (ML) models have leveraged existing large datasets to overcome issues of the physical-based modeling. They can establish the reliable predictive models for the classification of EMG signals. With interpretation of the intention from the human by the ML models, the control signals are inputted to robots to perform desired actions. Nevertheless, control of these devices via EMG remains incomplete and needs much improvement.

In this paper, we demonstrate the versatility of using minimized number of surface electrodes for collecting EMG signals, which can be classified and predicted by multiple real-time classifiers for controlling a robotic arm. The contribution of this work includes: (1) only two surface electrodes were employed (on each forearm) to reliably collect EMG signals that show ten classes of the hand motions; (2) two ML classifiers can real-time differentiate between the motions of the right-hand versus left-hand; (3) the Gaussian Naïve Bayes (GNB) classifier excels with only limited amounts of training data, and (4) combining both the ML classifiers into one output allows up to twenty five robotic commands from the five hand motions on each arm.

\section{RELATED WORK}

EMG signals have been extensively studied and used in both the academic and industrial fields. The first commercially available EMG system was launched in $1950 .{ }^{[11]}$ Since then, a variety types of systems have been introduced. EMG signals can be categorized into two main forms, the discreteaction EMG signals and the steady-state action EMG signals. The discrete motion EMG signals are generated by performing the quick actions to create the EMG waves, which is then analyzed and used to determine the motions. These waves are bell-shaped and analyzed in their entirety to determine the motion. In contrast the steady state EMG signals are generated by maintaining constant force during motions. The amplitudes of the steady state EMG signal are then used to determine the motions. The steady state EMG signals are more of a continuous waveform.

\subsection{Discrete-action EMG Signals: Measurement and Classification}

There has been much research in the discrete-action EMG signals. Young et. al. classified four types of discrete motions. ${ }^{[12]}$ Their motions included hand open/closed and wrist extension/flexion. They were able to accurately classify three out of these four motions. To achieve the function, their system used six pairs of active electrodes for non-amputees and eight pairs of active electrodes for the amputees. Huang et. al used the EMG signals in union with ultrasounds to classify fourteen discrete finger motions. ${ }^{[13]}$ They used eight active EMG electrodes on one forearm. The classifier classified the EMG signals with an accuracy of $>90 \%$ and the ultrasound signals with an accuracy of $>95 \%$. Krasoulis and 
Nazarpour used sixteen active electrodes to classify six finger motions on the participants. ${ }^{[14]}$ The participants were transracial amputees and were asked to perform imaginary finger movement using a prosthetic hand. The model achieved accuracies ranging from $74 \%$ to $82 \%$. Lu et. al used seven surface electrodes to classify six EMG motions for individuals with spinal cord injuries. ${ }^{[15]}$ The EMG signals from these motions were used to control a hand exoskeleton to help move the fingers of the participants. They achieved an average accuracy of $70 \%$. Martinez et. al tried to classify six types of hand motions. Their system used two Delsys EMG sensor bars on one arm ( 2 channels). The best classifier reached an accuracy of $93 \%$ on these six hand motions. ${ }^{[16]}$

\subsection{Steady State EMG Signals}

The steady state (SS) EMG signals have also been researched extensively. Crawford et. al developed a model for real-time classification of eight static hand motions using seven active electrodes on three subjects. ${ }^{[17]}$ The model achieved classification accuracy in between $92 \%$ and $98 \%$. Momen et. al allowed eight test participants to choose their own motions for classification of four-to-nine motions. ${ }^{[18]}$ On average, the participants were able to classify four motions at an accuracy of $80 \%$ or more. The EMG signals were collected from four active electrodes on the right arms. Their research showed that it was particularly important to choose motions with the EMG signals which can be differentiated by a ML algorithm. Lucas et. al used the SS EMG signals to control an exoskeleton to have the pinching motion of the index fingers and thumbs. ${ }^{[19]}$ Their system was only able to correctly detect the pinching motion in $67 \%$ of the tests. Matsubara et. al used four active electrodes to collect the EMG signals of five hand motions, which were then classified to control a robotic hand. ${ }^{[20]}$ The model achieved an accuracy of only $70 \%$.

While much research has been focused on the EMG signal collection and their real-time classification, the problem with the current research, however, is that the accurate signal collection requires a considerable number of active electrodes. For a practical application, collection of EMG signals for accurate classification from a minimized electrode is much needed to efficiently control robots. This current research is to tackle this issue. Herein, we demonstrate that with only two active electrodes on each forearm, five distinct hand motions on each arm can be classified by the sophisticated ML models. This ratio of number of hand motions to the number of the active electrodes is a considerable improvement over the previous reported systems. Furthermore, we show that the system allows for the real-time classification and motion control of a robotic hand from these EMG signals. Additionally, we show that a combination of two classifiers

Published by Sciedu Press with a single output allows for combination control, greatly increasing the number of available commands from minimal electrodes.

\section{EXPERIMENTAL DETAILS}

\subsection{System Design}

The individual began by performing one of the predetermined hand motions. The EMG signal was then generated from the forearm and recorded by surface electrodes which were connected to the Keithley DAQ6510. The waveform signal was recorded at a rate of 100 datapoints per second for 2 seconds using LabView. The waveform was then filtered and normalized to a value between 0 and 4 . This saved data was then sent to a Python script to train and test the Gaussian Naïve Bayes (GNB) classifier. The trained GNB classifier was then saved to another Python script. Then in real-time, new data was sent to the trained GNB python script for predicting the motions. Then the corresponding signal was sent to the raspberry pi $3 b$ to control the robotic arm in real-time. This flow of the EMG signal is shown in Figure 4a and illustrated in detail as follows.

To make the EMG signal applicable, the signal collection and processing in an efficient manner is critical, usually following the four steps. First, a raw EMG signal is recorded as a differential signal (the difference in voltage between the two surface electrodes) and then amplified. Second, the signal passes through a band pass filter to remove the low and high frequencies. Third, the signal is converted from analog to digital and then passes through a digital high-pass filter to further remove noise interference. Fourth, the signal is rectified and passes through a digital low-pass filter. The signal is again rectified by taking the absolute value of the signal. This rectified signal then passes through an additional digital low-pass filter. The additional digital low-pass filter can be applied in many ways such as taking the mean value or using a Butterworth filter. ${ }^{[21]}$

\subsection{Electrode Placement}

Our choice for electrode placement was determined through testing multiple locations on the arm with multiple motions. We tested the forearm, bicep, and triceps. We determined that the forearm was the best fitted for recording the motions of the hand. While the forearm is not solely responsible for controlling the hand, it provides the highest degree of freedom for the hand motions. This allows the GNB classifier to accurately determine the different motions. The motions that were chosen for our experiment are as follows: wrist flexion, wrist extension, wrist adduction, fist, and no motion for both hands. These motions were chosen because they produce EMG signals that are easily differentiated by the GNB 
classifier. These motions allow us to have eight movement commands and two stop commands. Each motion produces a different amplitude ranging from the lowest being no motion and the highest being wrist adduction. The amplitude is the important feature that was used by the GNB classifier to determine what motion is being performed. The GNB classifier will be explained in detail in the next section.

\subsection{Data Collection}

We collected data from 10 participants over the course of two session. We recorded each trial at a rate of 100 datapoints per second over the course of 2 seconds. We conducted 100 trials for each hand motion resulting in a total of 1,000 trials. We then used the training data from all motions to train our GNB classifier. Figure 2 shows the overall process of the data collection, preprocessing, and modeling training and testing based on the processed EMG signals. Our system's performance depends on multiple factors: (1) choosing appropriate motions that produce EMG signals that can be differentiated, (2) performing the motions in the same way every time (same distance of motion, same force applied, etc.).

\subsection{Gaussian Naïve Bayes Classification}

The GNB classifier is built off Bayes theorem. Bayes theorem is a mathematical model used to describe the probability that an event will occur given a set of data. The formula for the theorem is as follows:

$$
P_{A \mid B}=\frac{P_{B \mid A} * P_{A}}{P_{B}}
$$

where $P_{A \mid B}$ is the posterior probability of class $A$ given the predictor $B, P_{B \mid A}$ is the probability of predictor $B$ given class $A, P_{A}$ is the prior probability of class $A$, and $P_{B}$ is the prior probability of the predictor. Once the posterior probability is calculated for different classes, the class with the highest probability is selected. Training is fast because only the probability of each class and the probability of each class given different predictors are calculated.

For classification, if the number of trials in each class in the training data, then the probability of each class is equal. The GNB classifier is an extension of Naïve Bayes. For the GNB the Gaussian (or normal distribution) is used to estimate the distribution of the data. This is an easy estimation to make because to accomplish this, only the mean and standard deviation needs to be estimated from the training data. Prediction of new $x$ values is calculated using the Gaussian probability density function (PDF). When making the prediction, the new $x$ value, mean, and standard deviation are plugged into the Gaussian PDF which returns the probability that the new $x$ value is for the given class. The equation for the Gaussian PDF is as follows:

$$
P D F_{(x, \text { mean }, s d)}=\frac{1}{\sqrt{2 * P I} * s d} * \mathrm{e}^{-\frac{x-m e a n^{2}}{2 * s d^{2}}}
$$

where $s d$ is the standard deviation, $P I$ is the numerical constant, $x$ is the input value for the input variable, and e is the Euler's number.

We chose to use two GNB classifiers in real-time for two reasons. First, the right and left arm produce almost identical EMG signals for the same motions. This is due to the symmetry of the human body. Using two classifiers in real-time allows us to differentiate between the right and left fist, right and left wrist flexion, and so on. If there were only one classifier, there would be much misclassification for the same motions. Second, having two classifiers in real-time also eliminates cross talk interference between channels. This way, the right arm is independent of the left arm and vice versa. This allows only five motions to be chosen but allows the five motions to be ten motions in total in the classifier.

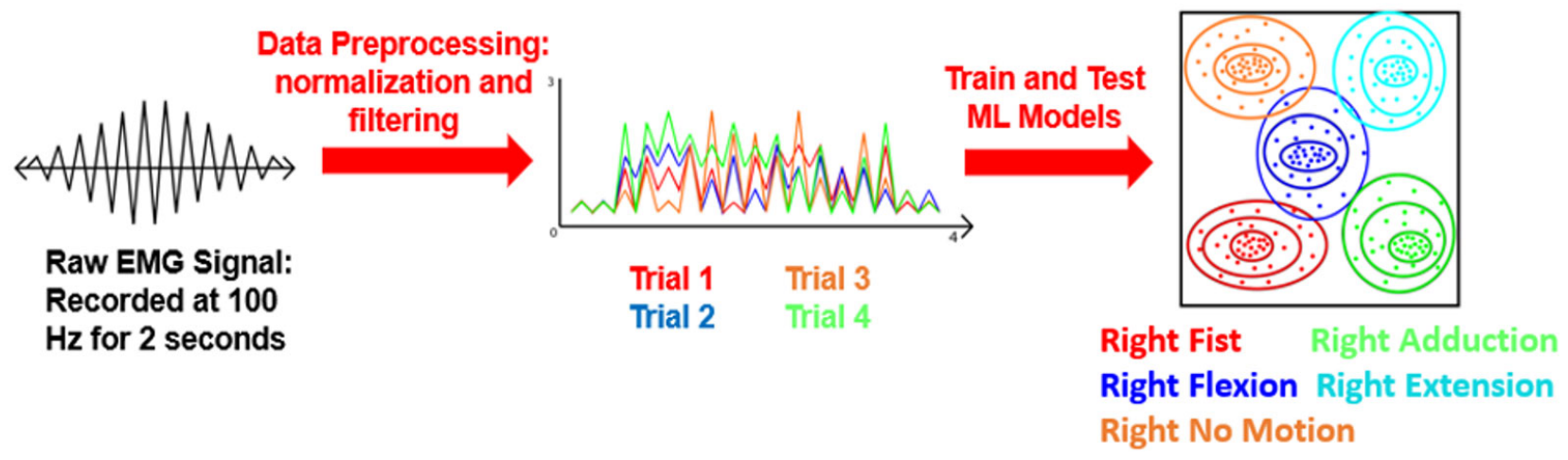

Figure 2. Overall process of data collection, preprocessing, and machine learning classification 


\section{Results AND Discussion}

\subsection{Classification of Hand Motions from EMG Signals by Machine Learning Algorithms}

We used the recorded data to train the GNB classifier. To do that, we performed five split k-fold cross validation. Figure 3 shows the training and testing accuracy of the model with the highest accuracy for each classifier that we tried on the right and left arms, respectively. Among the tested eight ML models, the GNB classifier achieves the highest testing accuracy of $>96 \%$ on both the right and left arms. Although some other models show a higher training accuracy, the GNB model has the highest testing accuracy without overfitting. The overfitting occurs when using the Random Forest, XGBoost, and Adaboost models, which is normally caused by
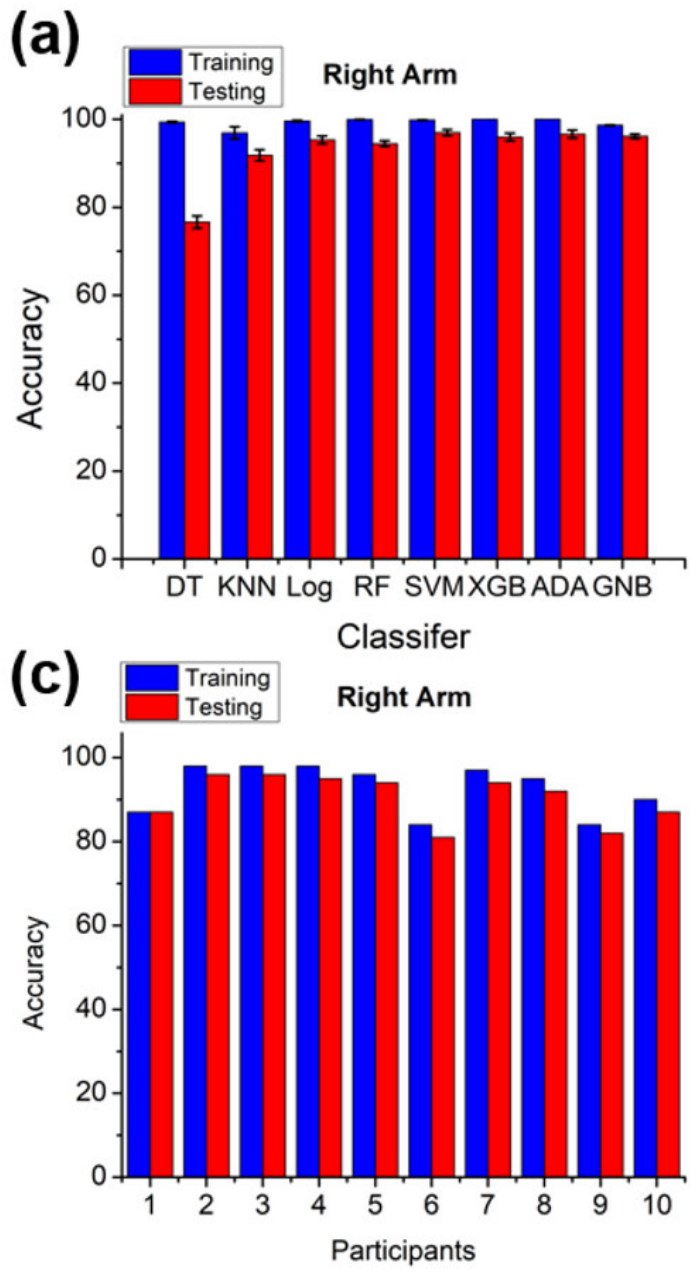

a lack of data. The GNB classifier does not show overfitting because the GNB classifier excels with small amount of training data. This is one of the reasons we chose to use the GNB classifier. Since we did not have an abundance of training data, the GNB worked perfectly. As shown in Figure $3 \mathrm{c}, \mathrm{d}$ among the tested ten participants, we achieved a testing accuracy of $>81 \%$ on the right arms and we achieved a testing accuracy of $>86 \%$ on the left arms except for Participant 6 and Participant 9 because some of their motions generated similar EMG waves. Averagely, we were able to obtain an accuracy of $92.9 \%$ for the testing data and $90.8 \%$ for the training data on the right arms as well as $89.7 \%$ for the training data and $87.2 \%$ for the testing data on the left arms.
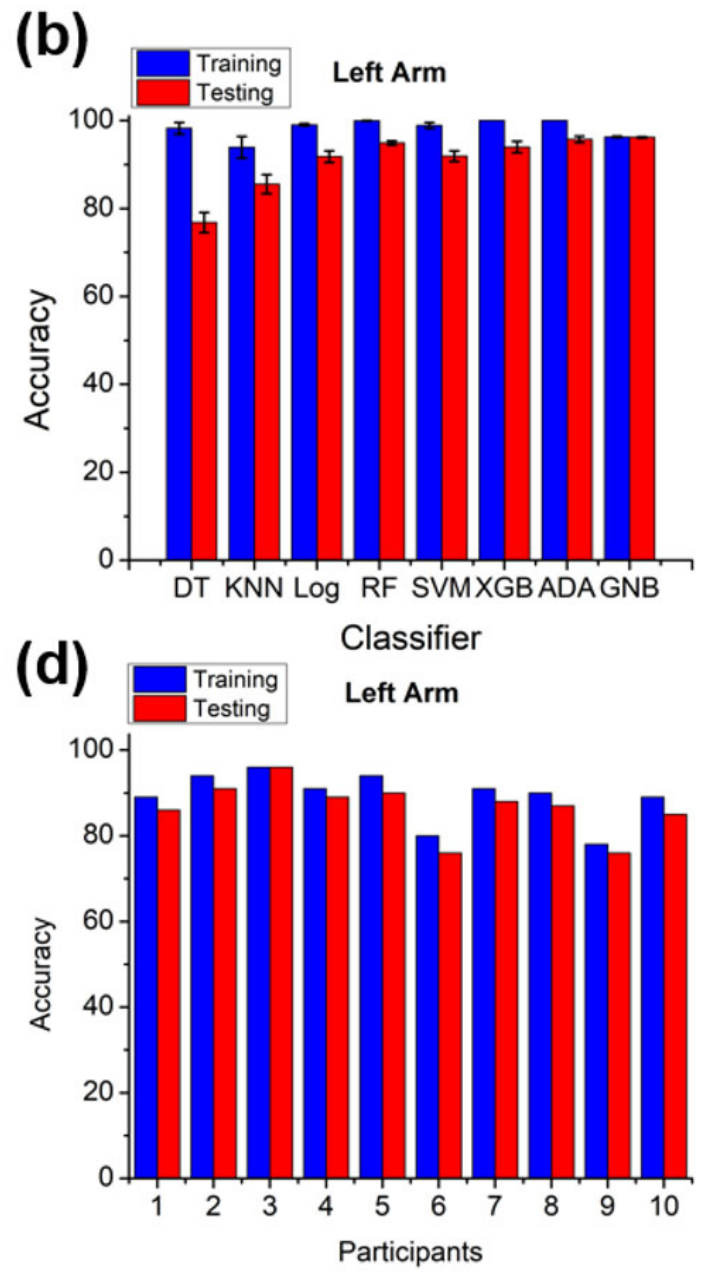

Figure 3. Training and testing accuracy of each classifier from data collected from right arms (a) and left arms (b). The classifiers from left to right are as follows: decision tree (DT), K-nearest neighbor (KNN), logistic regression (Log), random forest (RF), support vector machine (SVM), XGBoost (XGB), AdaBoost (ADA), and Gaussian Naïve Bayes (GNB). Training and testing accuracy of GNB classifier from data collected from right arms (c) and left arms (d) of each participant. 

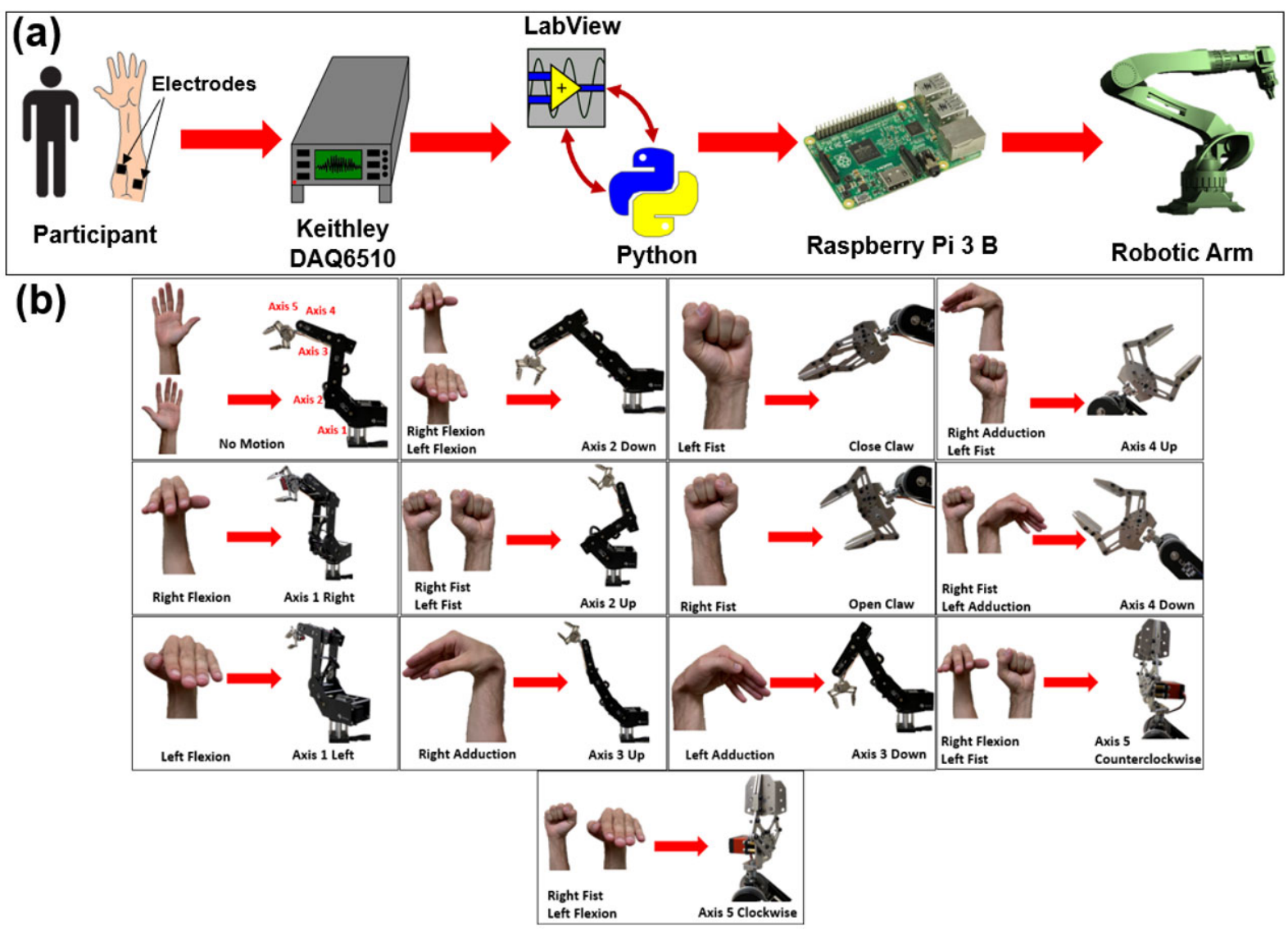

Figure 4. (a) Flow of EMG signal from participant to robotic arm. (b) Hand motions used and the corresponding motion of the robotic arm

\subsection{Real-time Control of a Robotic Arm by EMG Sig- nals}

The Dorna robotic arm consists of four axes and a gripper. Axis one moves the robotic arm right and left and is controlled by the right wrist flexion (for right) and the left wrist flexion (for left), respectively. Axis two moves the robotic arm up and down and is controlled by the right wrist flexion and the left wrist flexion (for down), and by the right hand fist and the left hand fist (for up), respectively. Axis three also moves up and down and is controlled by the right wrist adduction (for up) and the left wrist adduction (for down), respectively. Axis four rotates the robotic gripper clockwise (the right hand first and the left wrist flexion) and counterclockwise (the right wrist flexion and the left hand fist), respectively. Finally, axis five controls the gripper by opening and closing and is controlled by the right hand fist (to open the claw) and the left hand fist (to close the claw), respectively. These motions controlled by the EMG signals are shown in the supplementary video. To test the performance of our system, we used the GNB classifiers in real-time and attempted to perform tasks with the robotic arm. Please note that during the online experiments, the increase of the number of channels for recording data in real-time caused a second delay between the time the participants performed the motions, and the robotic arm performed the corresponding actions. To mitigate the problem, we first had the participants perform single action. We found that the participants were able to accurately perform eight out of the ten commands every time. An in-depth explanation of the problem and the solutions to the problem are explained as follows.

During the online portion of the experiment, the participants were able to accurately predict four out of five motions on both arms every time. The wrist extension motion that was more difficult to predict as it produced EMG signals with a low signal-to-noise ratio. Further the robotic arm caused noise interference, making the baseline signal higher and therefore, eliminating the range of the wrist extension motion. But we found a solution that greatly increased the level of accuracy that we could obtain. Our solution was to combine both predicted motions into one string which was sent to the robotic arm. The combination of hand motions was performed in the following ways. First, the participants performed hand motions with both the right and left hands, respectively; Second, each EMG signal was sent to a sepa- 
rate GNB classifier which predicted the hand motion being performed. As each predicted motion was programmed to a 5-to-7-character string (e.g., right fist and left wrist flexion was termed as RF_LWF), this string was then sent to the robotic arm to perform a corresponding motion. This allowed us to perform combinations of the four motions that were easily predicted. Using combinations, we now have a total of sixteen commands that can control the motions of the robot using only two pairs of active surface electrodes. Some combinations were not used to control the robotic arm. This was only due to the limited number of motions the robotic arm was capable of performing. During the course of recording training data, we also discovered that the size of the participants' arms also impacted the quality of the collected EMG signals. A larger area produced the better EMG signals. Nevertheless, these participants, were still able to easily record the tested motions.

Another issue we observed during the online experiments was the delay in the signal recording and processing. The data acquisition unit was about one to two seconds behind the participants. This caused the classifier to miss the ideal window of the EMG signals for proper classification. Increasing the speed of data recording would mitigate the issue. However, during the robotic control this would not have been an issue. The robotic arm would perform the first action it received after the stop command. The robotic arm would perform this action no matter what other actions it received until receiving the stop command. Once it received the stop command, it would stop and not move until it received another command.

The robotic arm in our experiment can perform 12 separate motions. However, these are the rigid motions that moved in one plane at a time. Recently, researchers have explored complex mathematical modeling to increase the efficiency of robotic manipulators. ${ }^{[22-25]}$ These models allow the robotic arms to follow complex motion paths. Currently, these models do not allow for real-time human intervention. Thus, we envision that integrating the EMG control with the complex mathematical modeling could allow for complex robotic motions under intervention of human. This integration would further increase the productivity of HRI in various application settings.

\section{Conclusion And Future Work}

We have shown that by combining the multiple EMG signals from minimal number of surface electrodes, we can maximize the amount of motions that can be accurately classified with ML models. We can accurately perform sixteen robotic commands using only four active electrodes (two channels). We presented an in-depth explanation of the GNB classifier and explained the necessity of having motions that produce the differentiable EMG signals. The highest classification accuracy of $>96 \%$ was obtained from the GNB classifier. Finally, we demonstrated that EMG signal could be used to real-time control a robotic arm.

In future, multiple biological signals can be extremely informative on the state and wellness of an individual. Information such as blood oxygen saturation, respiratory rate, heart rate, blood pressure, and temperature can determine if an individual is healthy or at risk of a health complication. Creating a system that can record and understand these signals would be beneficial to older adults or adults with certain health conditions. In order to understand these signals for classification, a robust ML model would need to be developed. These human-in-loop control algorithms would lead to collaborative robots that work side by side with humans.

\section{ACKNOWLEDGeMENTS}

This work was financially supported by US Department of Energy (Award number: DE-FE0031645).

\section{REFERENCES}

[1] Rai V, Sharma A, Rombokas E. Mode-free Control of Prosthetic Lower Limbs. In 2019 International Symposium on Medical Robotics (ISMR). 2019: 1-7. https ://dx .doi .org/10.1109/ISMR . 2019 .8710187

[2] Gao H, et al. EEG-Based Volitional Control of Prosthetic Legs for Walking in Different Terrains. IEEE Transactions on Automation Science and Engineering. 2019: 1-11. https://dx.doi.org/10. 1109/TASE. 2019. 2956110

[3] Abdhul AA, Subramani D, Ganesan J, et al. Design and Development of EMG Based Prosthetic Arm. In 2020 6th International Conference on Advanced Computing and Communication Systems (ICACCS), 6-7 March 2020 2020, pp. 502-504. https : //dx. doi.org/10.11

Published by Sciedu Press

\section{9/ICACCS48705.2020.9074206}

[4] Li G, Liu Y, Li Z. The Prosthetic Arm: A Dramatic Improvement For The Limb Amputation From The Humerus. In 2019 IEEE 4th International Conference on Advanced Robotics and Mechatronics (ICARM), 3-5 July 2019 2019, pp. 475-480. https : //dx. doi . o rg/10.1109/ICARM. 2019.8833890

[5] Jafarzadeh M, Hussey DC, Tadesse Y. Deep learning approach to control of prosthetic hands with electromyography signals. In 2019 IEEE International Symposium on Measurement and Control in Robotics (ISMCR). 2019: A1-4-1-A1-4-11. https ://dx.doi.org/10.11 09/ISMCR47492.2019.8955725

[6] Inhyuk M, Myungjoon L, Jeicheong R, et al. Intelligent robotic wheelchair with EMG-, gesture-, and voice-based interfaces. In Pro- 
ceedings 2003 IEEE/RSJ International Conference on Intelligent Robots and Systems (IROS 2003) (Cat. No.03CH37453). 27-31 Oct. 2003; 4: 3453-3458. https://dx.doi.org/10.1109/IROS. 20 03.1249690

[7] Inhyuk M, Myungjoon L, Junuk C, et al. Wearable EMG-based HCI for Electric-Powered Wheelchair Users with Motor Disabilities. In Proceedings of the 2005 IEEE International Conference on Robotics and Automation, 18-22 April 2005 2005, pp. 2649-2654. https://dx.doi.org/10.1109/ROBOT.2005.1570513

[8] Lenzi T, Rossi SMMD, Vitiello N, et al. Intention-Based EMG Control for Powered Exoskeletons. IEEE Transactions on Biomedical Engineering. 2012; 59(8): 2180-2190. https ://dx.doi.org/10. 1109/TBME. 2012.2198821

[9] Ao D, Song R, Gao J. Movement Performance of Human-Robot Cooperation Control Based on EMG-Driven Hill-Type and Proportional Models for an Ankle Power-Assist Exoskeleton Robot. IEEE Transactions on Neural Systems and Rehabilitation Engineering. 2017; 25(8): 1125-1134. https ://dx.doi .org/10.1109/TNSRE. 201 6.2583464

[10] Kiguchi K, Hayashi Y. An EMG-Based Control for an Upper-Limb Power-Assist Exoskeleton Robot. IEEE Transactions on Systems, Man, and Cybernetics, Part B (Cybernetics). 2012; 42(4): 1064-1071. https ://dx.doi.org/10.1109/TSMCB. 2012.2185843

[11] Ladegaard J. Story of electromyography equipment. Muscle Nerve Suppl. 2002; 11: S128-33. https://dx.doi.org/10.1002/mus .10176

[12] Young AJ, Smith LH, Rouse EJ, et al. Classification of simultaneous movements using surface EMG pattern recognition. IEEE Trans Biomed Eng. 2013; 60(5): 1250-8. https ://dx.doi .org/10.11 09/TBME. 2012. 2232293

[13] Huang Y, Yang X, Li Y, et al. Ultrasound-Based Sensing Models for Finger Motion Classification. IEEE J Biomed Health Inform. 2018; 22(5): 1395-1405. https://dx.doi.org/10.1109/JBHI . 2017 .2766249

[14] Krasoulis A, Nazarpour K. Discrete action control for prosthetic digits. bioRxiv, p. 2020.03.25.007203, 2020. https ://dx.doi.o $\mathrm{rg} / 10.1101 / 2020.03 .25 .007203$

[15] Lu Z, Stampas A, Francisco GE, et al. Offline and online myoelectric pattern recognition analysis and real-time control of a robotic hand after spinal cord injury. J Neural Eng. 2019; 16(3): 036018. https ://dx.doi.org/10.1088/1741-2552/ab0cf0

[16] Ramírez-Martínez D, Alfaro-Ponce M, Pogrebnyak O, et al. Hand Movement Classification Using Burg Reflection Coefficients. Sen- sors (Basel). 2019; 19(3). https://dx.doi.org/10.3390/s19 030475

[17] Crawford B, Miller K, Shenoy P, et al. Real-Time Classification of Electromyographic Signals for Robotic Control. vol. 2, ed. AAAI'05: Proceedings of the 20th national conference on Artificial intelligence, 2005, pp. 523-528.

[18] Momen K, Krishnan S, Chau T. Real-time classification of forearm electromyographic signals corresponding to user-selected intentional movements for multifunction prosthesis control. IEEE Trans Neural Syst Rehabil Eng. 2007; 15(4): 535-42. https ://dx.doi.org/10. 1109/TNSRE. 2007.908376

[19] Lucas L, DiCicco M, Matsuoka Y. An EMG-Controlled Hand Exoskeleton for Natural Pinching. J. Robotics Mechatronics. 2004; 16 : 482-488.

[20] Matsubara T, Hyon SH, Morimoto J. Learning and Adaptation of a Stylistic Myoelectric Interface: EMG-Based Robotic Control with Individual User Differences. 2011 IEEE International Conference on Robotics and Biomimetics: IEEE, 2011: 390-395.

[21] Chowdhury RH, Reaz MB, Ali MA, et al. Surface electromyography signal processing and classification techniques. Sensors (Basel). 2013 Sep; 13(9): 12431-66. https ://dx.doi.org/10.3390/s130912 431

[22] Zhang Z, Lin Y, Li S, et al. Tricriteria Optimization-Coordination Motion of Dual-Redundant-Robot Manipulators for Complex Path Planning. IEEE Transactions on Control Systems Technology. 2018; 26(4): 1345-1357. https://dx.doi.org/10.1109/TCST. 2017 .2709276

[23] Zhang Z, Chen S, Li S. Compatible Convex-Nonconvex Constrained QP-Based Dual Neural Networks for Motion Planning of Redundant Robot Manipulators. IEEE Transactions on Control Systems Technology. 2019; 27(3): 1250-1258. https ://dx.doi.org/10.1109 /TCST . 2018. 2799990

[24] Zhang Z, et al. A Varying-Parameter Convergent-Differential Neural Network for Solving Joint-Angular-Drift Problems of Redundant Robot Manipulators. IEEE/ASME Transactions on Mechatronics. 2018; 23(2): 679-689. https ://dx.doi.org/10.1109/TMECH. 2 018.2799724

[25] Zhang Z, Yan Z. A Varying Parameter Recurrent Neural Network for Solving Nonrepetitive Motion Problems of Redundant Robot Manipulators. IEEE Transactions on Control Systems Technology. 2019; 27(6): 2680-2687. https ://dx.doi.org/10.1109/TCST . 2018.2872471 